PROCEEDINGS OF THE

AMERICAN MATHEMATICAL SOCIETY

Volume 140, Number 8, August 2012, Pages 2903-2912

S 0002-9939(2011)11104-3

Article electronically published on November 28, 2011

\title{
A SIMPLE ALGEBRAIC CHARACTERIZATION OF NONSTANDARD EXTENSIONS
}

\author{
MARCO FORTI
}

(Communicated by Julia Knight)

\begin{abstract}
We introduce the notion of functional extension of a set $X$, by means of two natural algebraic properties of the operator "*" on unary functions. We study the connections with ultrapowers of structures with universe $X$, and we give a simple characterization of those functional extensions that correspond to limit ultrapower extensions. In particular we obtain a purely algebraic proof of Keisler's characterization of nonstandard (= complete elementary) extensions.
\end{abstract}

\section{INTRODUCTION}

Besides the "superstructure approach" proposed in [13] (see Section 4.4 of [5]), various presentations of the "nonstandard methods" that use nonstandard set theories have been started in [10] and [8] and are continuously developing. However it seems that, in some sense, the nonstandard methods do not really need such set-theoretic generality. In fact, several different "elementary" approaches are also available, starting from the very interesting one of [9] (see also [1]). A general "algebraic" characterization of nonstandard extensions is given by W. S. Hatcher in [7. Another purely algebraic construction producing every nonstandard model is proposed in 2. A survey 4 of such "elementary introductions" to nonstandard methods has been jointly presented by V. Benci, M. Di Nasso, and the author.

This paper originates from a reflection on the topological approach to nonstandard models exploited in [3, 6]. There a nonstandard extension of a set $X$ is considered as a sort of "topological completion" ${ }^{*} X$, where $X$ is a discrete dense subspace and each function $f: X \rightarrow X$ has a continuous extension ${ }^{*} f:{ }^{*} X \rightarrow{ }^{*} X$. In the Hausdorff case of [3], these extensions are uniquely determined by the topology. Since these nonstandard models turned out to be a very narrow class of spaces, more general $T_{1}$-spaces are considered in [6]. Uniqueness of continuous extensions of functions being thus lost, the " $*$ " operator was changed to provide a distinguished continuous extension to ${ }^{*} X$ of each function $f: X \rightarrow X$. As a consequence, in this general case the topology does not force the choice of the continuous extensions, but it is rather this choice that induces a topology on ${ }^{*} X$. These considerations suggest that one might find purely "algebraic" conditions on the $*$-extensions of functions, so as to characterize all nonstandard extensions, without any mention of topologies.

Received by the editors December 24, 2010 and, in revised form, January 31, 2011 and March 3, 2011.

2010 Mathematics Subject Classification. Primary 03H05, 03C07, 03C20; Secondary 26E35.

This work was partially supported by MIUR Grants PRIN 2007, 2009, Italy.

(C)2011 American Mathematical Society 
In this paper we consider simple supersets ${ }^{*} X$ of $X$ together with an operator $*$ : $X^{X} \rightarrow{ }^{*} X^{*} X$, which provides a distinguished extension of each function $f: X \rightarrow X$. We show that three algebraic conditions on the " $*$ " operator are all that is needed to make such a functional extension a true nonstandard model of $X$, and this in a very simple and natural way. Our main result is the following.

Main Theorem. Let ${ }^{*} X$ be a proper superset of the set $X$, and assume that to every function $f: X \rightarrow X$ is associated a distinguished extension ${ }^{*} f:{ }^{*} X \rightarrow{ }^{*} X$ satisfying the following conditions:

(comp) ${ }^{*} g \circ{ }^{*} f={ }^{*}(g \circ f)$, for all $f, g: X \rightarrow X$;

(diag) let $\chi_{\Delta}$ be a 0-1-valued binary function such that $\chi_{\Delta}(x, y)=1$ if and only if $x=y$, for any relevant elements $x$ and $y$. Then

$$
{ }^{*}\left(\chi_{\Delta}(f(\cdot), g(\cdot))(\xi)=\chi_{\Delta}\left({ }^{*} f(\xi),{ }^{*} g(\xi)\right)\right.
$$

for all $f, g: X \rightarrow X$ and all $\xi \in{ }^{*} X$;

(dir) for all $\xi, \eta \in{ }^{*} X$ there exist $f, g: X \rightarrow X$ and $\zeta \in{ }^{*} X$ such that ${ }^{*} f(\zeta)=\xi$ and ${ }^{*} g(\zeta)=\eta$.

Then there exist an ultrafilter $\mathcal{D}$ over $I={ }^{*} X \times X$ and a filter $\mathcal{E}$ of equivalences on I such that the structure ${ }^{*} \mathfrak{X}=\left\langle{ }^{*} X ;\left\{{ }^{*} f \mid f \in X^{X}\right\}\right\rangle$ is isomorphic to the limit ultrapower $\mathfrak{X}_{\mathcal{D}}^{I} \mid \mathcal{E}=\left\langle X_{\mathcal{D}}^{I} \mid \mathcal{E} ;\left\{\bar{f} \mid f \in X^{X}\right\}\right\rangle$ of the structure $\mathfrak{X}=\left\langle X ;\left\{f \mid f \in X^{X}\right\}\right\rangle$.

So ${ }^{*} \mathfrak{X}$ is a nonstandard extension of $\mathfrak{X}$.

For precise definitions of the involved notions we refer to the next section. Here we simply anticipate that, as is apparent for (comp) and (diag), also the third condition (dir) admits a (stronger) first-order formulation. So, on the one hand, our criterion generalizes and greatly simplifies that of [7. On the other hand, by directly defining the ultrafilter $\mathcal{D}$ and the equivalences $\mathcal{E}$, it provides a new, "purely algebraic" proof of the celebrated Keisler characterization of complete elementary extensions (see Theorem 6.4.10 of [5]). To be sure, the definitions of $I, \mathcal{D}$, and $\mathcal{E}$ depend on the given extension ${ }^{*} \mathfrak{X}$, so we do not provide a new construction of nonstandard extensions, but rather a simple, easily verifiable characterization of all these structures. We hope that this axiomatic approach to nonstandard methods may be of independent interest.

The paper is organized as follows. In Section 1 we give the formal definition of functional extensions and we study the first "preservation properties" of these extensions. In Section 2, we prove the main theorem and obtain our "functional" characterization of nonstandard (= complete elementary) extensions. Concluding remarks can be found in the final Section 3, where in particular we briefly outline the connections between functional extensions and the topological extensions of [6].

In general, we refer to [5] for definitions and facts concerning ultrapowers, ultrafilters, and nonstandard models that are used in this paper.

\section{Functional extensions}

A main feature of all nonstandard models of analysis is the existence of a canonical extension ${ }^{*} f:{ }^{*} \mathbb{R} \rightarrow{ }^{*} \mathbb{R}$ of any (standard) function $f: \mathbb{R} \rightarrow \mathbb{R}$ (and also of any subset $A \subseteq \mathbb{R}$ ). Here we use this property as the definition of functional extensions of an arbitrary set $X$. We shall assume in the sequel that $0,1 \in X$, in order to have at our disposal the extensions of characteristic functions. Since the extension 
of the characteristic function of $A \subseteq X$ will turn out to be a characteristic function in ${ }^{*} X$, we shall use it to define the extension ${ }^{*} A$ of $A$ in ${ }^{*} X$.

The so-called nonstandard methods are intended to study extensions which preserve those properties of the standard structure which are currently being considered. The Transfer (Leibniz's) Principle states that all properties that are expressible in a(n) (in)sufficiently expressive language are preserved by passing to the nonstandard models. In particular, an important property of nonstandard models of analysis is that "disjoint functions have disjoint extensions". This property has a clear "analytic" flavour, and in fact it can be considered as the most characteristic feature of nonstandard extensions when compared with continuous extensions of functions in compactifications or topological completions, where equality may be reached at limit points only (see [6]). So we should reasonably postulate this property, together with the preservation of characteristic functions.

With this in mind, in the following definition we assume that "composition and diagonal are preserved".

Definition 1.1. A proper superset ${ }^{*} X$ of the set $X$ is a functional extension of $X$ if to every function $f: X \rightarrow X$ is associated a distinguished extension ${ }^{*} f:{ }^{*} X \rightarrow{ }^{*} X$ in such a way that the following conditions are fulfilled, for all $f, g: X \rightarrow X$ and all $\xi \in{ }^{*} X$ :

(comp) ${ }^{*} g\left({ }^{*} f(\xi)\right)=*(g \circ f)(\xi)$, and

(diag) ${ }^{*}\left(\chi_{\Delta}(f(\cdot), g(\cdot))(\xi)=\chi_{\Delta}\left({ }^{*} f(\xi),{ }^{*} g(\xi)\right)\right.$, where $\chi_{\Delta}$ is a 0 -1-valued binary function such that $\chi_{\Delta}(x, y)=1 \Longleftrightarrow x=y$.

The functional extension ${ }^{*} X$ of $X$ is directed if

(dir) for all $\xi, \eta \in{ }^{*} X$ there exist $f, g: X \rightarrow X$ and $\zeta \in{ }^{*} X$ such that ${ }^{*} f(\zeta)=\xi$ and ${ }^{*} g(\zeta)=\eta$.

Following the common usage, we call standard the points of $X$ and nonstandard those of * $X \backslash X$. The adjective "directed" refers to the so-called Puritz order, a preordering of nonstandard models corresponding to the Rudin-Keisler (pre)ordering of ultrafilters (see [12, 11]):

for $\xi, \eta \in{ }^{*} X$ put $\eta \leq_{P} \xi$ if there exists $f: X \rightarrow X$ such that $\eta={ }^{*} f(\xi)$.

Then clearly the property (dir) expresses that the Puritz order $\leq_{P}$ on ${ }^{*} X$ is directed.

We are interested only in directed extensions, but all functional extensions satisfy various natural "preservation properties". We begin by considering characteristic functions, so as to define the extensions of subsets and derive their properties.

Theorem 1.2. Let ${ }^{*} X$ be a functional extension of $X$. Then

(i) if $c_{x}: X \rightarrow X$ is the constant function with value $x \in X$, then the extension ${ }^{*} c_{x}$ is the constant with value $x$ on ${ }^{*} X$;

(ii) if $\chi_{A}: X \rightarrow X$ is the characteristic function of $A \subseteq X$, then the extension ${ }^{*} \chi_{A}$ is the characteristic function of a superset ${ }^{*} A$ of $A$ in ${ }^{*} X$.

(iii) The map ${ }^{*}: A \mapsto{ }^{*} A$ commutes with binary union, intersection and complement. Moreover ${ }^{*} A \cap X=A$; hence ${ }^{*}$ is a Boolean isomorphism of $\mathcal{P}(X)$ onto a subfield $S t\left({ }^{*} X\right)$ of $\mathcal{P}\left({ }^{*} X\right)$.

Proof. Since $c_{1}=\chi_{\Delta}(f(\cdot), f(\cdot))$, the property (diag) implies that ${ }^{*} c_{1}$ is the constant 1 on ${ }^{*} X$. Any other constant is obtained from $c_{1}$ by composing with a suitable transposition, and so (i) follows from (comp). 
Moreover $\chi_{A}=\chi_{\Delta}\left(\chi_{A}(\cdot), c_{1}(\cdot)\right)$; hence ${ }^{*} \chi_{A}$ takes only the values 0 and 1 . Since obviously ${ }^{*} \chi_{A}$ maps $A$ to 1 , we have (ii).

Let $\tau$ be a transposition mapping 0 to some $x \neq 0,1$. Then $*\left(\tau \circ \chi_{B}\right)$ is 1 exactly on ${ }^{*} B$, and so ${ }^{*}\left(\chi_{A \cap B}\right)={ }^{*}\left(\chi_{\Delta}\left(\chi_{A}(\cdot), \tau\left(\chi_{B}(\cdot)\right)\right)\right)=\chi^{*} A \cap{ }^{*} B$. So the map ${ }^{*}$ commutes with binary intersection. Moreover $\chi_{\Delta}\left(\chi_{A}(x), \chi_{X \backslash A}(x)\right)=0$ for all $x \in X$; hence ${ }^{*} A$ and ${ }^{*}(X \backslash A)$ give a partition of ${ }^{*} X$. So we have at once that ${ }^{*} A \cap X=A$ and that ${ }^{*} X \backslash{ }^{*} A={ }^{*}(X \backslash A)$. It follows that the map * is a Boolean isomorphism, and (iii) is proved.

Having defined $*$-extensions of sets, the property (diag) gives immediately a sort of "preservation of equalizers", which corresponds to another basic idea of nonstandard analysis, namely that "standard functions behave like germs":

Corollary 1.3. Let ${ }^{*} X$ be a functional extension of $X$. Then

$$
\left\{\xi \in{ }^{*} X \mid{ }^{*} f(\xi)={ }^{*} g(\xi)\right\}={ }^{*}\{x \in X \mid f(x)=g(x)\}
$$

for all $f, g: X \rightarrow X$, or equivalently, for all $\xi \in{ }^{*} X$,

$$
{ }^{*} f(\xi)={ }^{*} g(\xi) \Longleftrightarrow \exists A \subseteq X .\left(\xi \in{ }^{*} A \quad \& \forall x \in A . f(x)=g(x)\right) .
$$

It follows from Theorem 1.2 and Corollary 1.3 that ${ }^{*} X$ induces a uniquely determined functional extension ${ }^{*} A$ of any subset $A \subseteq X$. Namely, for $f: A \rightarrow A$, let ${ }^{*} f:{ }^{*} A \rightarrow{ }^{*} A$ be the restriction to ${ }^{*} A$ of ${ }^{*} g$, where $g: X \rightarrow X$ is any function whose restriction to $A$ is $f$.

Notice that the identity map may not be preserved by functional extensions. If $\imath: X \rightarrow X$ is the identity of $X$, in the general case one only obtains

$$
{ }^{*} f(\xi)={ }^{*} f\left({ }^{*} \imath(\xi)\right)={ }^{*} \imath\left({ }^{*} f(\xi)\right) \text { for all } f: X \rightarrow X \text { and all } \xi \in{ }^{*} X .
$$

Thus ${ }^{*} l$ is the identity exactly on those points of ${ }^{*} X$ that are reached by some function ${ }^{*} f$, and all functions ${ }^{*} f$ map each nonstandard point $\xi$ to the same point as ${ }^{*}(\xi)$. So, when ${ }^{*} \imath$ is not the identity, the extension ${ }^{*} X$ can be considered "redundant", in the sense that the extensions of all functions are completely determined by their restrictions to ${ }^{*}\left({ }^{*} X\right)$, and the remaining elements of ${ }^{*} X$ are not attained by any function ${ }^{*} f$. Moreover ${ }^{*} \imath\left({ }^{*} X\right)$, equipped with the restrictions of all ${ }^{*} f \mathrm{~s}$, becomes a functional extension where the identity is preserved.

Call irredundant a functional extension ${ }^{*} X$ of $X$ if the following weakening of (dir) holds:

for all $\xi \in{ }^{*} X$ there exist $f: X \rightarrow X$ and $\eta \in{ }^{*} X$ such that ${ }^{*} f(\eta)=\xi$.

Important and natural preservation properties, concerning ranges, injectivity, and finite subsets can be derived for irredundant extensions, hence a fortiori for directed extensions:

Proposition 1.4. Let ${ }^{*} X$ be an irredundant functional extension of $X$. Then, for all $f: X \rightarrow X$ and all $A \subseteq X$,

(i) ${ }^{*} f\left({ }^{*} A\right)={ }^{*}(f(A))$ (in particular ${ }^{*} f$ is onto if $f$ is onto);

(ii) if $f: X \rightarrow X$ is one-one on $A$, then ${ }^{*} f$ is one-one on ${ }^{*} A$.

(iii) Extensions of finite sets are trivial; i.e. $A={ }^{*} A$ whenever $A$ is finite.

Proof. In irredundant extensions ${ }^{*} \imath$ is the identity. Hence $(i)$ and (ii) follow from (comp), because (the restriction of) a function is injective (resp. surjective) if and only if it has a left (resp. right) inverse. 
In order to obtain $($ iii $)$ observe first that ${ }^{*} \chi_{\{1\}}={ }^{*}\left(\chi_{\Delta}\left(\imath(\cdot), c_{1}(\cdot)\right)\right)$ is 1 exactly on 1. Hence ${ }^{*}\{1\}=\{1\}$, and the same property holds for all singletons, by point $(i)$. Since ${ }^{*}$ is a Boolean isomorphism, all of (iii) follows.

In order to make an effective use of nonstandard models, it is always assumed by nonstandard analysts that all and only infinite sets are indeed extended; i.e. $A={ }^{*} A$ if and only if $A$ is finite. We shall not need this assumption, but all properties of Proposition 1.4 are valid in any nonstandard model. So we have in mind essentially only irredundant extensions. We have not postulated irredundancy in Definition 1.1 because this condition is still too weak to obtain fully nonstandard extensions. To this aim we have isolated in the Introduction the stronger property (dir) that "every pair of points is dominated by some point".

Remark that all the properties stated in Theorem 1.2 and its corollaries, as well as the defining properties (comp) and (diag), are particular (and perspicuous) cases of the Transfer Principle. On the other hand, this seems prima facie not to apply to the condition (dir), whose straightforward formalization is second-order. On the contrary, a strong uniform version of directedness can be obtained as an instance of transfer. Namely one can compose any bijective function $\delta: X \rightarrow X \times X$ with the ordinary projections $\pi_{i}: X \times X \rightarrow X$ so as to obtain "unary projections" $p_{1}, p_{2}: X \rightarrow X$ such that

for all $x, y \in X$ there is a unique $z \in X$ such that $p_{1}(z)=x, p_{2}(z)=y$.

Then by Transfer one obtains the following strengthening of (dir):

$$
\text { for all } \xi, \eta \in{ }^{*} X \text { there is a unique } \zeta \in{ }^{*} X \text { such that }{ }^{*} p_{1}(\zeta)=\xi,{ }^{*} p_{2}(\zeta)=\eta \text {. }
$$

So, if we want a full Transfer Principle in our functional extensions, then not only irredundancy but also directedness has to hold at the end. On the other hand, the very point of this paper is the remarkable fact that we shall prove in the next section:

- by combining the sole property (dir) with the simple, natural conditions (comp) and (diag), one forces the strongest Transfer Principle for all first-order properties, thus providing complete elementary extensions.

\section{Algebraic CHARACTERIZATION OF DiRECTED FUNCTIONAL EXTENSIONS}

We devote this section to a "purely algebraic" proof of the Main Theorem.

Recall that two functions $f, g: I \rightarrow X$ are equivalent modulo $\mathcal{U}$, where $\mathcal{U}$ is an arbitrary ultrafilter over $I$, if they agree on some set $U \in \mathcal{U}$. The ultrapower $X_{\mathcal{U}}^{I}$ is the set of the equivalence classes modulo $\mathcal{U}$ of all functions $f: I \rightarrow X$. We refer to [5] for basic facts about ultrapowers. In the sequel, in dealing with ultrapowers, we shall adhere to the following notation:

- $[f] \in X_{\mathcal{U}}^{I}$ is the equivalence class of the function $f: I \rightarrow X$;

- $\bar{g}: X_{\mathcal{U}}^{I} \rightarrow X_{\mathcal{U}}^{I}$ is the interpretation of the function $g: X \rightarrow X$ in the ultrapower, i.e. $\bar{g}([f])=[g \circ f]$ for all $f: I \rightarrow X$;

- $A^{I} / \mathcal{U} \subseteq X_{\mathcal{U}}^{I}$ is the interpretation of $A \subseteq X$ in the ultrapower.

The limit ultrapower $X_{\mathcal{U}}^{I} \mid \mathcal{E}$, where $\mathcal{U}$ is an ultrafilter over $I$ and $\mathcal{E}$ is a filter of equivalences on $I$, is the subset of the ultrapower $X_{\mathcal{U}}^{I}$ containing the $\mathcal{U}$-equivalence classes of all functions $f: I \rightarrow X$ that induce on $I$ an equivalence

$$
E q(f)=\{(x, y) \mid f(x)=f(y)\}
$$


that belongs to $\mathcal{E}$. (Notice that one can assume w.l.o.g. that each $E \in \mathcal{E}$ gives a partition of size not exceeding $|X|$.)

A celebrated theorem of Keisler's states that every complete elementary extension 11 of a structure $\mathfrak{X}$ with universe $X$ is isomorphic to a limit ultrapower of $X$ (see e.g. Section 6.4 of [5]). We shall obtain the same conclusion from the much weaker assumption of the three properties (dir), (comp), and (diag).

Starting from a given directed functional extension ${ }^{*} X$ of $X$, we now define a limit ultrapower $X_{\mathcal{D}}^{I} \mid \mathcal{E}$ that will end up being isomorphic to ${ }^{*} X$.

- For $\alpha \in{ }^{*} X$ put $\check{\alpha}=\left\{\xi \in{ }^{*} X \mid \exists f: X \rightarrow X\right.$ s.t. $\left.\alpha={ }^{*} f(\xi)\right\}$, and for all $\xi \in \check{\alpha}$ fix a function $f_{\xi \alpha}: X \rightarrow X$ such that ${ }^{*} f_{\xi \alpha}(\xi)=\alpha$.

- The family $\left\{\check{\alpha} \mid \alpha \in{ }^{*} X\right\}$ has the finite intersection property, because ${ }^{*} X$ is directed, and so we can extend it to an ultrafilter over ${ }^{*} X$, say $\mathcal{V}$.

- Put $I={ }^{*} X \times X$. For $\alpha \in{ }^{*} X$, define the function

$$
\widehat{\alpha}: I \rightarrow X \quad \text { by } \quad \widehat{\alpha}(\xi, x)= \begin{cases}f_{\xi \alpha}(x) & \text { if } \xi \in \check{\alpha} \\ x & \text { otherwise }\end{cases}
$$

and the corresponding equivalence $E_{\alpha}$ induced on $I$ by

$$
E_{\alpha}=E q(\widehat{\alpha})=\{(i, j) \in I \times I \mid \widehat{\alpha}(i)=\widehat{\alpha}(j)\} .
$$

- Let $\mathcal{U}_{\xi}=\left\{A \subseteq X \mid \xi \in{ }^{*} A\right\}$ be the ultrafilter on $X$ generated by $\xi$, and let $\mathcal{D}=\sum_{\mathcal{V}} \mathcal{U}_{\xi}$ be the ultrafilter on $I$ such that

$$
D \in \mathcal{D} \Longleftrightarrow\left\{\xi \in{ }^{*} X \mid\{x \in X \mid(\xi, x) \in D\} \in \mathcal{U}_{\xi}\right\} \in \mathcal{V} .
$$

- For $\alpha \in{ }^{*} X$ and $D \in \mathcal{D}$ put

$$
E_{\alpha}^{D}=E_{\alpha} \cap D \times D \cup i d_{I}=\{(i, j) \in D \times D \mid \widehat{\alpha}(i)=\widehat{\alpha}(j)\} \cup\{(i, i) \mid i \in I \backslash D\} .
$$

Then we have

Lemma 2.1. For all $\alpha, \beta \in{ }^{*} X$ and all $g: X \rightarrow X$,

$$
\beta={ }^{*} g(\alpha) \Longleftrightarrow[\widehat{\beta}]=[g \circ \widehat{\alpha}](\bmod \mathcal{D}) .
$$

It follows that the family of equivalences

$$
\left\{E_{\alpha}^{D} \mid \alpha \in^{*} X, D \in \mathcal{D}\right\}
$$

has the finite intersection property.

Proof. Assume $\beta={ }^{*} g(\alpha)$. Then, for all $\xi \in \check{\alpha}$,

$$
{ }^{*} f_{\xi \beta}(\xi)=\beta={ }^{*} g(\alpha)=\left({ }^{*} g \circ{ }^{*} f_{\xi \alpha}\right)(\xi) .
$$

Applying Corollary 1.3 we obtain, for all $\xi \in \check{\alpha}$,

$$
\mathcal{U}_{\xi} \ni\left\{x \in X \mid\left(g \circ f_{\xi \alpha}\right)(x)=f_{\xi \beta}(x)\right\}=\{x \in X \mid g(\widehat{\alpha}(\xi, x))=\widehat{\beta}(\xi, x)\} .
$$

Hence $\{i \in I \mid g(\widehat{\alpha}(i))=\widehat{\beta}(i)\} \in \mathcal{D}$, i.e. $[\widehat{\beta}]=[g \circ \widehat{\alpha}]$.

Conversely, assume $\{i \in I \mid g(\widehat{\alpha}(i))=\widehat{\beta}(i)\} \in \mathcal{D}$. Then

$$
\begin{aligned}
\mathcal{V} \ni & \left\{\xi \in \check{\alpha} \mid\{x \mid g(\widehat{\alpha}(\xi, x))=\widehat{\beta}(\xi, x)\} \in \mathcal{U}_{\xi}\right\} \\
& =\left\{\xi \in \check{\alpha} \mid\left[f_{\xi \beta}\right]=\left[g \circ f_{\xi \alpha}\right] \bmod \mathcal{U}_{\xi}\right\}=\left\{\xi \in \check{\alpha} \mid{ }^{*} f_{\xi \beta}(\xi)={ }^{*} g\left({ }^{*} f_{\xi \alpha}(\xi)\right\} .\right.
\end{aligned}
$$

Therefore $\beta={ }^{*} g(\alpha)$.

\footnotetext{
${ }^{1}$ That is, an extension that satisfies the Transfer Principle w.r.t. a language containing all $n$-ary relations over $X$.
} 
Now let $E_{\beta}^{G}, E_{\gamma}^{H}$ be given, and pick $\alpha, g, h$ such that $\beta={ }^{*} g(\alpha)$ and $\gamma={ }^{*} h(\alpha)$. Then there exists $D \subseteq G \cap H$ in $\mathcal{D}$ such that $\widehat{\beta}, \widehat{\gamma}$ agree respectively with $g \circ \widehat{\alpha}, h \circ \widehat{\alpha}$ on $D$. Hence $E_{\alpha}^{D} \subseteq E_{\beta}^{G} \cap E_{\gamma}^{H}$.

Let $\mathcal{E}$ be the filter of equivalences generated by the family

$$
\left\{E_{\alpha}^{D} \mid \alpha \in{ }^{*} X, D \in \mathcal{D}\right\} .
$$

We are ready to state

Theorem 2.2. Let ${ }^{*} X$ be a directed functional extension of $X$. Then the map

$$
\text { ^: }{ }^{*} X \rightarrow X^{I}
$$

induces an isomorphism of ${ }^{*} \mathfrak{X}=\left\langle{ }^{*} X ;\left\{{ }^{*} f \mid f \in X^{X}\right\}\right\rangle$ onto the limit ultrapower $\mathfrak{X}_{\mathcal{D}}^{I} \mid \mathcal{E}=\left\langle X_{\mathcal{D}}^{I} \mid \mathcal{E} ;\left\{\bar{f} \mid f \in X^{X}\right\}\right\rangle$ (as structures for the language $\mathcal{L}=\{f \mid f: X \rightarrow$ $X\})$.

Proof. By taking $g=i d$ in Lemma 2.1 we obtain that the map ${ }^{\wedge}$ is injective modulo $\mathcal{D}$. Moreover the map ${ }^{*} g$ is transformed in $\bar{g}$; hence ${ }^{-}$preserves extensions of functions. So we have only to prove that the range of $\widehat{ }$ contains a representative of the class modulo $\mathcal{D}$ of every function $\varphi: I \rightarrow X$ such that $E q(\varphi) \in \mathcal{E}$.

Let $\varphi: I \rightarrow X$ be such a function. Then there exist $\alpha \in{ }^{*} X$ and $D \in \mathcal{D}$ such that $E_{\alpha}^{D} \subseteq E q(\varphi)$; i.e., $\varphi$ is constant on each equivalence class modulo $E_{\alpha}^{D}$. Assume w.l.o.g. that $D \subseteq \check{\alpha} \times X$. Then $\varphi$ is completely determined by its behaviour on a set including representatives for all classes of $\check{\alpha} \times X$, which may be conveniently chosen as $\{(\alpha, x) \mid x \in X\}$.

Put $g(x)=\varphi(\alpha, x)$ and $\beta={ }^{*} g(\alpha)$. By Lemma 2.1 we have

$$
\widehat{\beta}(\alpha, x)=\widehat{{ }^{*} g(\alpha)}(\alpha, x)=(g \circ \widehat{\alpha})(\alpha, x)=g(x) .
$$

Hence $[\varphi]=[\widehat{\beta}]$.

According to the above theorem, any directed functional extension is a limit ultrapower, which in turn is a complete elementary extension. So we have the following corollary, which incorporates Keisler's Theorem:

Corollary 2.3. The following conditions are equivalent for any functional extension ${ }^{*} X$ of $X$ :

(1) ${ }^{*} X$ is directed;

(2) ${ }^{*} X$ is a limit ultrapower of $X$;

(3) ${ }^{*} X$ can be uniquely expanded to a nonstandard (complete elementary) extension of $X$.

In the next section we shall sketch two different proofs, a "topological" and a "purely logical" one, of the implication $(1) \Rightarrow(3)$ in the above corollary, i.e. the "logical part" of our Main Theorem. To be sure, in order to completely prove Corollary 2.3, either of these proofs has to be combined with Keisler's Theorem.

\section{Concluding Remarks}

We conclude this paper by outlining two different approaches, a "topological" one and a "purely logical" approach. Each of them can provide a proof that directed functional extensions are complete elementary extensions. 
3.1. The star topology of directed functional extensions. We pointed out in the Introduction that this paper has been inspired by the topological approach to nonstandard models presented in [6], where the extended functions ${ }^{*} f$ are taken to be continuous (and ${ }^{*} A$ is the closure in ${ }^{*} X$ of $A \subseteq X$ ). However, while in the case of the Hausdorff extensions of [3], the topology uniquely determines the extension, on the contrary, in the general case it seems that the "algebraic" properties of the map * are responsible for the topology rather than vice versa. Let us report the following definition from [ $[$ :

Definition 3.1. A $T_{1}$ topological space ${ }^{*} X$ is a topological extension of $X$ if

- $X$ is a dense subspace of ${ }^{*} X$, and

- to every function $f: X \rightarrow X$ is associated a distinguished continuous extension ${ }^{*} f:{ }^{*} X \rightarrow{ }^{*} X$ in such a way that

(c) ${ }^{*} g \circ{ }^{*} f={ }^{*}(g \circ f)$ for all $f, g: X \rightarrow X$, and

(i) if $f(x)=x$ for all $x \in A \subseteq X$, then ${ }^{*} f(\xi)=\xi$ for all $\xi \in \bar{A}$.

- The topological extension ${ }^{*} X$ is analytic if, for all $f, g: X \rightarrow X$,

(d) $f(x) \neq g(x)$ for all $x \in X \Longrightarrow{ }^{*} f(\xi) \neq{ }^{*} g(\xi)$ for all $\xi \in{ }^{*} X$.

- The topological extension ${ }^{*} X$ is coherent if

(f) for all $\xi, \eta \in{ }^{*} X$ there exist functions $p, q: X \rightarrow X$ and a point $\zeta \in{ }^{*} X$ such that ${ }^{*} p(\zeta)=\xi$ and ${ }^{*} q(\zeta)=\eta$.

It is easily seen that (i) and (d) follow from (diag). So all properties (cidf) of the above definition hold in any directed functional extension. As a matter of fact, it is this definition that suggested the defining properties (comp, diag, dir) assumed in this paper. So it seems natural to look for a topology that turns any directed functional extension into a coherent analytic extension.

In fact this task has been accomplished already in [6], where it is shown how to put on every nonstandard model ${ }^{*} X$ a natural topology, named star topology, that makes all functions ${ }^{*} f$ continuous. The closed sets of this topology are the arbitrary intersections of sets of the form

$$
E(\vec{f} ; \vec{\eta})=E\left(f_{1}, \ldots, f_{n} ; \eta_{1}, \ldots, \eta_{n}\right)=\left\{\xi \in{ }^{*} X \mid \exists i \in\{1, \ldots, n\} \cdot{ }^{*} f_{i}(\xi)=\eta_{i}\right\}
$$

for all $n$-tuples of functions $f_{i}: X \rightarrow X$, and of points $\eta_{i} \in{ }^{*} X$.

It is easily seen that the star topology is the coarsest $T_{1}$ topology on ${ }^{*} X$ that makes all functions ${ }^{*} f$ continuous, and in fact we have

Theorem 3.2. Every directed functional extension ${ }^{*} X$ of $X$, when endowed with the star topology, becomes a coherent analytic topological extension of $X$.

Proof. We have already remarked that the properties (cidf) are derivable from (comp, diag, dir). So we have only to prove that $X$ is dense in ${ }^{*} X$ w.r.t. the star topology. Assume that $X \subseteq E(\vec{f}, \vec{\eta})$. Since only nonstandard elements can be mapped to nonstandard elements by functions ${ }^{*} f$, we may assume w.l.o.g. that $\eta_{i}=y_{i} \in X$ for $i=1, \ldots, n$.

Then

$$
E(\vec{f}, \vec{y})=\bigcup_{i}^{*} f_{i}^{-1}\left(y_{i}\right)=\bigcup_{i} *\left(f_{i}^{-1}\left(y_{i}\right)\right)=*\left(\bigcup_{i} f_{i}^{-1}\left(y_{i}\right)\right) .
$$

It follows that $X=E(\vec{f}, \vec{y}) \cap X=\bigcup_{i} f_{i}^{-1}\left(y_{i}\right)$, and so ${ }^{*} X=E(\vec{f}, \vec{y})=\bar{X}$. 
Now Theorem 5.5 of [ $[$ states that all coherent analytic extensions are complete elementary extensions. Thus, if we had been ready to accept the full topological machinery of [6], we could have obtained Theorem 2.2 by combining the above theorem with Keisler's Theorem. But the aim of this paper is rather that of showing that a few clear, natural, purely algebraic conditions are all that is needed to obtain limit ultrapowers, hence full nonstandard models.

3.2. An inductive logical proof. The very reason for considering only unary functions in the definition of functional extensions is the fact that the properties (comp, diag, dir) together allow for a unique unambiguous definition of the extension * $\varphi$ of any $n$-ary function $\varphi: X^{n} \rightarrow X$ in a simple "parametric" way.

Theorem 3.3. Let ${ }^{*} X$ be a directed functional extension of $X$. Then there is a unique way of assigning an extension ${ }^{*} \varphi$ to every n-ary function $\varphi: X^{n} \rightarrow X$ so as to preserve all compositions 2 namely

$$
{ }^{*} \varphi\left(\xi_{1}, \ldots, \xi_{n}\right)={ }^{*}\left(\varphi \circ\left(f_{1}, \ldots, f_{n}\right)\right)(\zeta),
$$

where $f_{i}: X \rightarrow X$ and $\zeta \in{ }^{*} X$ are such that ${ }^{*} f_{i}(\zeta)=\xi_{i}$ for $i=1, \ldots, n$.

Proof. We can easily generalize the property (dir) and prove by induction on $n$ that, for all $\xi_{1}, \ldots, \xi_{n} \in{ }^{*} X$ there exist $f_{1}, \ldots, f_{n}: X \rightarrow X$ and $\zeta \in{ }^{*} X$ such that ${ }^{*} f_{i}(\zeta)=\xi_{i}$ for $i=1, \ldots, n$.

When the extensions of $n$-ary functions preserve compositions, the equality

$$
{ }^{*} \varphi\left(\xi_{1}, \ldots, \xi_{n}\right)={ }^{*}\left(\varphi \circ\left(p_{1}, \ldots, p_{n}\right)\right)(\zeta)
$$

has to hold whenever $\xi_{1}, \ldots, \xi_{n}, f_{1}, \ldots, f_{n}$, and $\zeta$ satisfy the above conditions. Therefore the extensions of unary functions completely determine those of all $n$ ary functions.

We are left with the task of showing that this definition is independent of the choice of the functions $f_{i}$ and of the point $\zeta$. Thus one has to prove

$$
{ }^{*}\left(\varphi \circ\left(f_{1}, \ldots, f_{n}\right)\right)(\xi)={ }^{*}\left(\varphi \circ\left(g_{1}, \ldots, g_{n}\right)\right)(\eta)
$$

for all $\varphi: X^{n} \rightarrow X$, provided $f_{1}, \ldots, f_{n}, g_{1}, \ldots, g_{n}: X \rightarrow X$ and $\xi, \eta \in{ }^{*} X$ satisfy ${ }^{*} f_{i}(\xi)={ }^{*} g_{i}(\eta)$ for $i=1, \ldots, n$.

Taking a point $\zeta$ such that ${ }^{*} p_{1}(\zeta)=\xi$ and ${ }^{*} p_{2}(\zeta)=\eta$, we can assume w.l.o.g. that $\xi=\eta=\zeta$. Then, applying Corollary 1.3, we get

$$
\begin{aligned}
& \left\{\xi \in{ }^{*} X \mid{ }^{*}\left(\varphi \circ\left(f_{1}, \ldots, f_{n}\right)\right)(\xi)={ }^{*}\left(\varphi \circ\left(g_{1}, \ldots, g_{n}\right)\right)(\xi)\right\}= \\
& *\left\{x \in X \mid\left(\varphi \circ\left(f_{1}, \ldots, f_{n}\right)\right)(x)=\left(\varphi \circ\left(g_{1}, \ldots, g_{n}\right)\right)(x)\right\} \supseteq \\
& \bigcap_{1 \leq i \leq n}{ }^{*}\left\{x \in X \mid f_{i}(x)=g_{i}(x)\right\}=\bigcap_{1 \leq i \leq n}\left\{\xi \in{ }^{*} X \mid{ }^{*} f_{i}(\xi)={ }^{*} g_{i}(\xi)\right\},
\end{aligned}
$$

and the proof is complete.

By using the characteristic functions in $n$ variables one can assign an extension ${ }^{*} R$ also to each $n$-ary relation $R$ on $X$. In this way, given a complete first-order structure $\mathfrak{X}=\left\langle X ; R_{i}, i \in I ; F_{j}, j \in J\right\rangle$ and a directed functional extension ${ }^{*} X$ of its universe, one produces a similar structure ${ }^{*} \mathfrak{X}=\left\langle{ }^{*} X ;{ }^{*} R_{i}, i \in I ;{ }^{*} F_{j}, j \in J\right\rangle$ with universe ${ }^{*} X$.

\footnotetext{
${ }^{2}$ That is, for all all $m, n \geq 1$, all $\varphi: X^{n} \rightarrow X$, and all $\psi_{1}, \ldots, \psi_{n}: X^{m} \rightarrow X$, ${ }^{*} \varphi \circ\left({ }^{*} \psi_{1}, \ldots,{ }^{*} \psi_{n}\right)={ }^{*}\left(\varphi \circ\left(\psi_{1}, \ldots, \psi_{n}\right)\right)$.
} 
Now, in order to obtain a "logical" proof that $\mathfrak{X}$ is an elementary substructure of $* \mathfrak{X}$, one should prove, for every formula $\Phi$, that

$$
\forall x_{1}, \ldots, x_{n} \in X .{ }^{*} \mathfrak{X} \models \Phi\left[x_{1}, \ldots, x_{n}\right] \Longleftrightarrow \mathfrak{X} \models \Phi\left[x_{1}, \ldots, x_{n}\right] .
$$

The atomic case being dealt with in Theorem 3.3, one could proceed, as usual, by induction on the complexity of the formula $\Phi$. But developing the details of such an inductive proof lies outside the scope of this paper.

\section{ACKNOWLEDGMENT}

The author is grateful to Mauro Di Nasso and Vieri Benci for useful discussions and suggestions. The author also thanks the referee for some useful remarks and chiefly for detecting a mistake in a previous version of the proof of Theorem 2.2.

\section{REFERENCES}

1. V. Benci, M. Di Nasso, Alpha-theory: an elementary axiomatics for nonstandard analysis, Expo. Math. 21 (2003), 355-386. MR2022004 (2004i:03108)

2. V. Benci, M. Di Nasso, A purely algebraic characterization of the hyperreal numbers, Proc. Amer. Math. Soc. 133 (2005), 2501-2505. MR2146232 (2006b:26036)

3. V. Benci, M. Di Nasso, M. Forti, Hausdorff nonstandard extensions, Boletim Soc. Parana. Mat. (3) 20 (2002), 9-20. MR2010860 (2004h:54019)

4. V. Benci, M. Di Nasso, M. Forti, The Eightfold Path to Nonstandard Analysis, in Nonstandard Methods and Applications in Mathematics (N.J. Cutland, M. Di Nasso, D.A. Ross, eds.), Lecture Notes in Logic 25, Assoc. Symbol. Logic, 2006, 3-44. MR2209073 (2006m:26066)

5. C.C. Chang, H.J. Keisler, Model Theory (3rd edition), North-Holland, Amsterdam, 1990. MR.1059055 (91c:03026)

6. M. Di Nasso, M. Forti, Topological and nonstandard extensions, Monatshefte für Mathematik 144 (2005), 89-112. MR2123958 (2005j:03050)

7. W.S. Hatcher, Elementary extension and the hyperreal numbers, in Mathematical Logic and Formal Systems, Lecture Notes in Pure Appl. Math., Dekker, New York, 1985, 205-219. MR 801912 (87b:03147)

8. K. Hrbàček, Axiomatic foundations for nonstandard analysis, Fund. Math. 98 (1978), 1-19. MR:528351 (84b:03084)

9. H.J. Keisler, Foundations of Infinitesimal Calculus, Prindle, Weber and Schmidt, Boston, 1976.

10. E. Nelson, Internal set theory: a new approach to nonstandard analysis, Bull. Amer. Math. Soc. 83 (1977), 1165-1198. MR0469763 (57:9544)

11. S.-A. Ng, H. Render, The Puritz order and its relationship to the Rudin-Keisler order, in Reuniting the antipodes - Constructive and nonstandard views of the continuum (P. Schuster, U. Berger, H. Osswald, eds.), Kluwer AP, Dordrecht, 2001, 157-166. MR.1895391 (2003d:54045)

12. C. Puritz, Ultrafilters and standard functions in non-standard arithmetic, Proc. London Math. Soc. (3) 22 (1971), 705-733. MR0289283 (44:6474)

13. A. Robinson, E. Zakon, A set-theoretical characterization of enlargements, in Applications of Model Theory to Algebra, Analysis and Probability (W.A.J. Luxemburg, ed.), Holt, Rinehart and Winston, New York, 1969, 109-122. MR0239965 (39:1319)

Dipartimento di Matematica Applicata "U. Dini", Universitá di Pisa, Via Buonarroti 1C, 56100 Pisa, Italy 